\title{
Diphtheria outbreak among the Rohingya refugees in Bangladesh: What strategies should be utilized for prevention and control?
}

\author{
Kamrul Hsan ${ }^{\mathrm{a}, \mathrm{b}}$, Jannatul Mawa Misti ${ }^{\mathrm{c}, \mathrm{d}}$, David Gozal ${ }^{\mathrm{e}}$, Mark D. Griffiths ${ }^{\mathrm{f}}$ and Mohammed A. Mamun $^{\mathrm{a}, \mathrm{c}}$ \\ ${ }^{a}$ Department of Public Health and Informatics, Jahangirnagar University, Savar, Dhaka-1342, \\ Bangladesh \\ ${ }^{\mathrm{b}}$ Institute of Allergy and Clinical Immunology of Bangladesh, Savar, Dhaka-1341, Bangladesh \\ ${ }^{\mathrm{c}}$ Undergraduate Research Organization, Savar, Dhaka, Bangladesh \\ ${ }^{\mathrm{d}}$ Pabna Medical College, Pabna, Bangladesh \\ ${ }^{\mathrm{e}}$ Department of Child Health and the Child Health Research Institute, The University of \\ Missouri School of Medicine, Columbia, USA \\ f International Gaming Research Unit, Psychology Department, Nottingham Trent University, \\ UK
}

Corresponding author-

\section{M.A. Mamun}

Director, Undergraduate Research Organization, Gerua Rd, Savar, Dhaka-1342, Bangladesh.

E-mail: mamunphi46@gmail.com, Mobile: +8801738592653. ORCID: https://orcid.org/00000002-1728-8966 


\section{Title: Diphtheria outbreak among the Rohingya refugees in Bangladesh: What strategies should be utilized for prevention and control?}

Keywords: Diphtheria; Outbreak; Control and prevention; Rohingya; Bangladesh.

\section{Dear Sir,}

The Rohingya refugees from Myanmar are one of the most ill-treated and vulnerable refugee groups in the world. This has led to an influx of 919,000 to Bangladesh. This influx has created extensive pressure in the refugee camps and essential services including food, water, health resources, shelter, and sanitation are in poor supply.

Studies show poor living conditions in refugee settings, along with infectious disease-mediating factors (i.e., no access to healthcare services, low sanitation and hygiene, etc.) leads to increase of diseases including diphtheria etc. ${ }^{[1,2]}$. Diphtheria is one of the most worrying infectious diseases particularly when routine childhood immunization programs are virtually non-existent ${ }^{[1,2]}$. Diphtheria affects the upper respiratory tract and is common among young children ${ }^{[3]}$. It may spread through symptomatic/asymptomatic carriers, leading to life-threatening complications and death, mostly in non-immunized children ${ }^{[1,3]}$. Since November 2017, diphtheria outbreaks among the refugees have become a public health crisis with the initial index case reported on November $10^{\text {th }}, 2017$, in the Balukhali camp ${ }^{[1]}$.

Just one month after reporting the first case (November, 2017), there were 440 additional suspected cases of diphtheria reported in neighboring refugee settlements, with 168 new cases reported on a single day (December 9, 2017) ${ }^{[1]}$. By December 2017, there had been 28 deaths and 3,014 suspected cases $^{[4]}$. By January 2018, 4907 diphtheria cases had been reported (35 deaths), with an incidence rate of 50-60 daily cases from 18-27 January $2018^{[4]}$. However, the number of suspected cases increased to 8,339 by the end of $2018^{[4]}$. Of these, 290 case-patients had tested positive using the Polymerase chain reaction (PCR) test, with 2,710 being classified as probable, and 5,339 being classified as suspected ${ }^{[4]}$.

By March 29, 2019, the total number of diphtheria case-patients was 8,487 in the Early Warning, Alert and Response System (EWARS) where 2,726 were probable and 5,483 were suspected ${ }^{[4]}$. $67 \%$ of cases were $<15$ years-old, and $13 \%$ younger than 5 years ( $56 \%$ females). The vast majority were not vaccinated against any diseases, due to limited healthcare in Myanmar. Furthermore, 202 diphtheria case-patients were reported from the host community surrounding the camps. Of the 208 cases, 30 were confirmed (PCR test), 65 probable, and 113 suspected $^{[5]}$.

Three rounds of vaccination campaigns were carried out among the Rohingya refugees (December 2017-March 2018), targeting children $<15$ years and a diphtheria vaccination campaign was also carried out January-February 2018. Additionally, the campaign included a booster dose of Penta/Td targeting children 1-15 years-of-age. Consequently, the incidence rate decreased, but presence of total cases is still high (Figure 1). The present outbreak demonstrates the potential for more severe outbreaks since the population has both large numbers of non-immune adults and unvaccinated children. Moreover, it also spreading among the nearby host community which may 
spread to another region of Bangladesh. Therefore, appropriate control and prevention strategies are needed to reduce the spread of the outbreak.

Previously, in the newly independent states (NIS) of the former Soviet Union and Baltic States, the World Health Organization (WHO)/United Nation Children Emergency Fund (UNICEF) strategy of 'one dose for all' was successful in boosting immunity among all age groups to high levels ${ }^{[3]}$. In the early years of diphtheria epidemics (among NIS population), investigation and control measures failed to control the spread of diphtheria until immunization campaigns closed the population immunity gap. In contrast, an outbreak of diphtheria in Mongolia in 1994-1995 was rapidly controlled by mass immunization ${ }^{[3]}$. Studies also show that incidence rates decrease quickly among countries achieving high immunization coverage among adults (e.g., Moldova where a successful mass campaign in summer 1995 leading to no winter peak occurrence and the epidemic was stopped $)^{[3]}$.

Overall, the rapid one-dose strategy (one dose of diphtheria and tetanus toxoid, with at least two limits of flocculation diphtheria toxoid per dose) was appropriate and effective in the NIS. It is especially suitable for diphtheria outbreaks and epidemics involving large populations where it is not possible to quickly provide the amount of vaccine needed for a multi-dose strategy. Although several immunization programs have been launched in camps, vaccination coverage was still low (only $22 \%$ ) - especially among adults ${ }^{[8]}$. Consequently, the one-dose strategy would be a better choice to control the diphtheria outbreak among the Rohingya refugees and host communities. Early detection and proper management of diphtheria cases and close contacts also aligns with the WHO/UNICEF strategy ${ }^{[3]}$. Treatment should start immediately, if doctor suspects diphtheria, and they should not wait for confirmatory laboratory tests. Those who have had contact with diphtheria cases should be monitored for development of disease, have specimens collected, be treated with antibiotics, and should be given a full course of diphtheria vaccine it they are not vaccinated. Contacts who test positive should be monitored until two subsequent cultures become negative after treatment.

The implementation of these prevention and control measures for both Rohingya refugees and host community requires large-scale international assistance to mobilize material resources. The process requires close collaboration between the health authorities of host countries and international agencies. In addition, coordinated planning and action from the existing humanitarian agencies are needed to ensure an effective EWARS, and improved treatment facilities, as well as management of diphtheria cases and their close contacts. International agencies should be supplied with additional diagnostic kits and other laboratory support to help overcome ongoing severe limitations in basic laboratory capacity. Additionally, the health sectors of Bangladesh should rationalize and consolidate services to reduce duplication of health service and ensure appropriate geographic distribution in health facilities. 


\section{References}

[1] Finger F, Funk S, White K, Siddiqui R, Edmunds WJ, Kucharski AJ. Real-time analysis of the diphtheria outbreak in forcibly displaced Myanmar nationals in Bangladesh. BMC medicine 2019,17(1),58.

[2] Eiset AH, Wejse C. Review of infectious diseases in refugees and asylum seekers - current status and going forward. Public Health Reviews 2017;38(1):22.

[3] Dittmann S, Wharton M, Vitek C, et al. Successful control of epidemic diphtheria in the states of the Former Union of Soviet Socialist Republics: lessons learned. Journal of Infectious Diseases 2000;181(Supplement_1):S10-22.

[4] Reliefweb. Bangladesh: Diphtheria outbreak - Dec 2017 ongoing. 2019. https://reliefweb.int/disaster/ep-2017-000177-bgd (accessed April 7, 2019).

[5] World Health Organization. Emergency: Rohingya Crisis - WHO Bangladesh Bi-weekly Situation Report \# 06. 29 March. 2019. http://www.searo.who.int/bangladesh/biweeklysitrep06cxbban2019.pdf (accessed April 7, 2019).

[6] World Health Organization. Emergency type: Rohingya Refugee Crisis - WHO Bangladesh Weekly Situation Report \# 41. $30 \quad$ August. 2018. http://www.searo.who.int/bangladesh/weeklysitrep41cxbban.pdf (accessed April 7, 2019).

[7] World Health Organiation. Emergency type: Rohingya Refugee Crisis - WHO Bangladesh Weekly Situation Report \# 45. 4 October. 2018. http://www.searo.who.int/bangladesh/weeklysitrep45cxbban.pdf?ua=1\&ua=1 (accessed April 7, 2019).

[8] World Health Organization. Emergency type: Rohingya Refugee Crisis - WHO Bangladesh Bi-weekly Situation Report \# $01 . \quad 17 \quad$ January 2019. http://www.searo.who.int/bangladesh/bi-weeklysitrep01 cxbban.pdf?ua=1\&ua=1 (accessed April 7, 2019). 
Table: Cases of Diphtheria among Rohingya refugees ${ }^{4,6,7}$

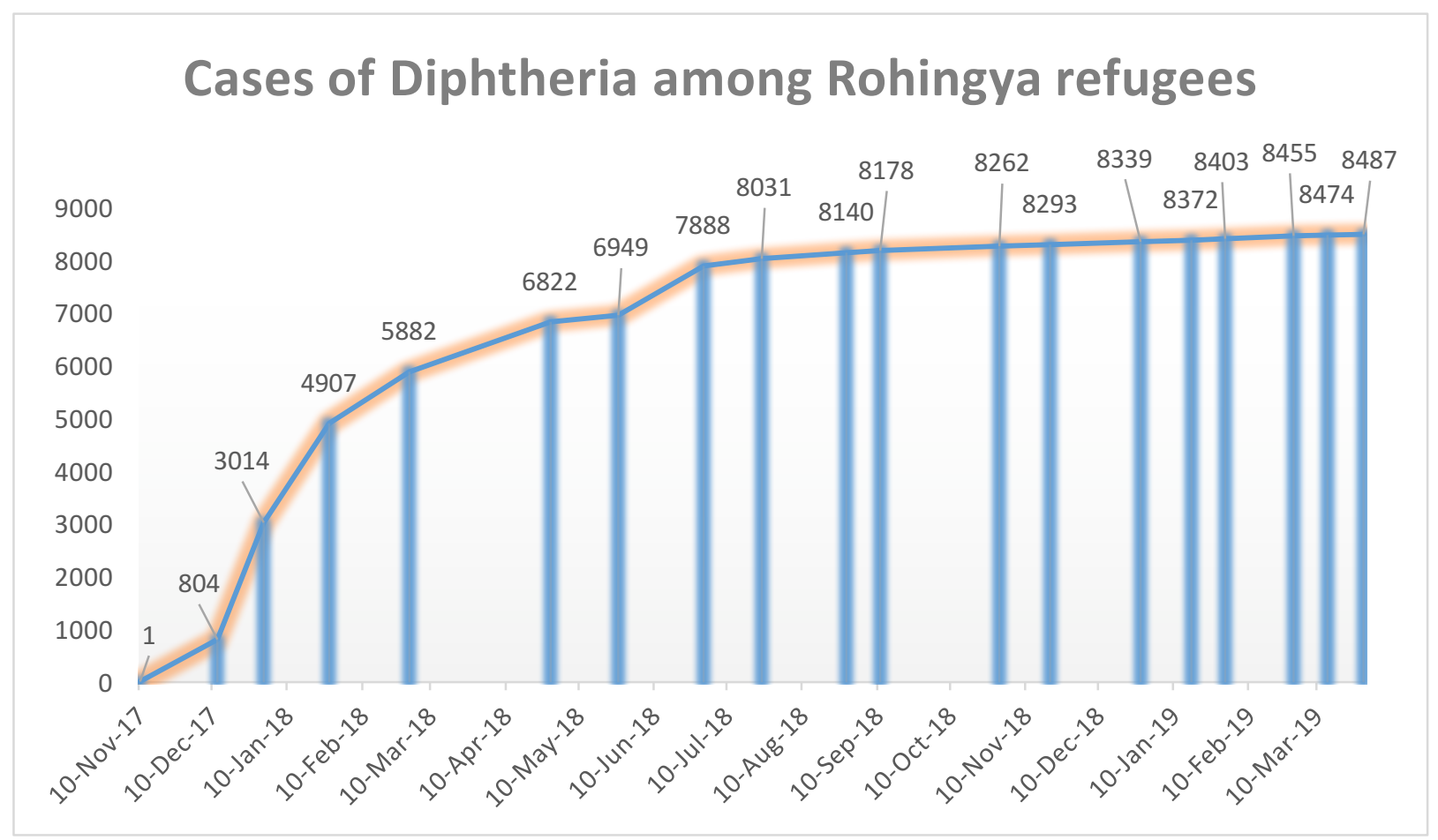

